\title{
LA-UR-21-22890
}

Approved for public release; distribution is unlimited.

Title:

Author(s):

Intended for:

Issued:
ERDE: THE EARTH-FIELD RESONANCE DETECTION AND EVALUATION DEVICES

Kaseman, Derrick

Web

2021-03-24 
Disclaimer:

Los Alamos National Laboratory, an affirmative action/equal opportunity employer, is operated by Triad National Security, LLC for the National Nuclear Security Administration of U.S. Department of Energy under contract 89233218CNA000001. By approving this article, the publisher recognizes that the U.S. Government retains nonexclusive, royalty-free license to publish or reproduce the published form of this contribution, or to allow others to do so, for U.S. Government purposes. Los Alamos National Laboratory requests that the publisher identify this article as work performed under the auspices of the U.S. Department of Energy. Los Alamos National Laboratory strongly supports academic freedom and a researcher's right to publish; as an institution, however, the Laboratory does not endorse the viewpoint of a publication or guarantee its technical correctness. 


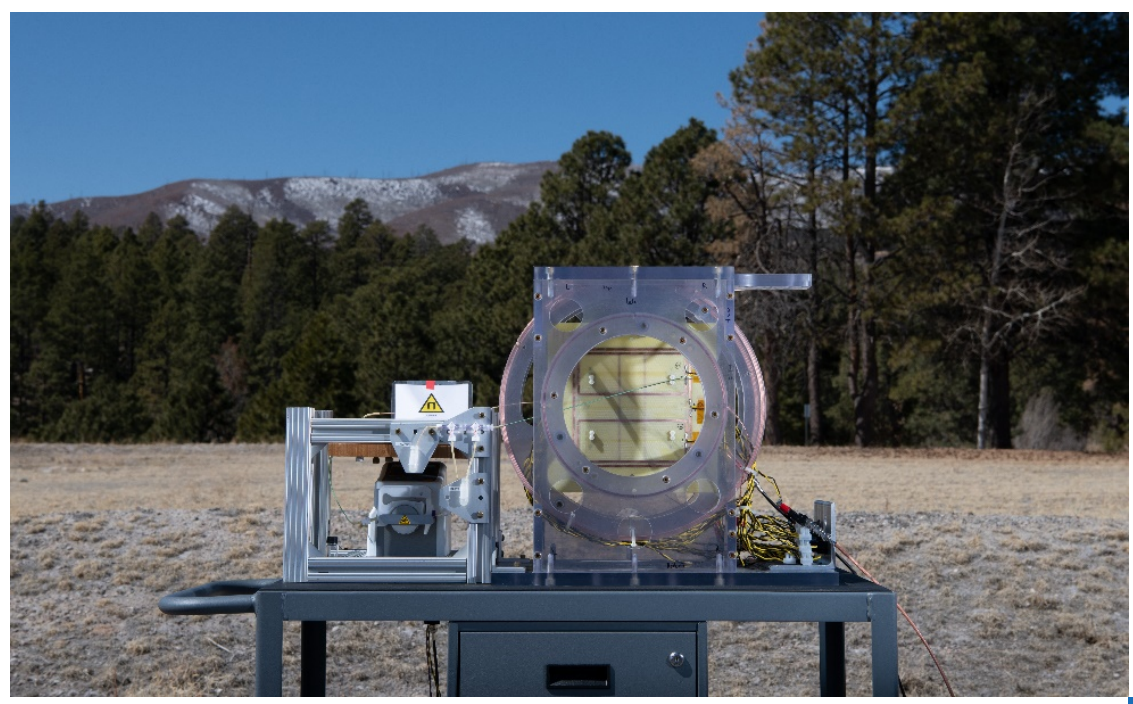

\section{ERDE: THE EARTH-FIELD RESONANCE DETECTION AND EVALUATION DEVICES}

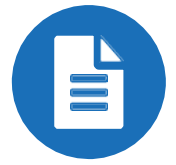

\section{SUMMARY}

Researchers at Los Alamos have developed the Earth-field Resonance Detection and Evaluation devices, or ERDE (German for "Earth") which brings portable chemical analysis and identification into the field. ERDE uses the Earth's magnetic field to perform nuclear magnetic resonance measurements without the need for sample preparation, costly cryogens, or calibration procedures, meaning it is extremely userfriendly. In fact, using the Earth's magnetic field allows detection and identification of chemical species through non-magnetic pipes without sampling, reducing the likelihood of costly biofouling. This innovative new device brings together these outstanding benefits to improve processes in the detection of noxious chemicals in water supplies as well as the identification of toxic materials anywhere. There are two fully-functional ERDE prototypes and efforts are underway to miniaturize the system further for improved portability, increased sensitivity, and expanded capabilities. The Laboratory is seeking qualified licensing or CRADA partners to finalize development of this system.

\section{MARKET APPLICATION}

ERDE lends outstanding accuracy without the need to sample, eliminating the problem of biofouling while giving chemical identification capabilities to the user. ERDE has many potential applications within chemical manufacturing and pharmaceutical industry quality control. Both require the identification and assurance of chemical species being manufactured. The pharmaceutical quality control industry alone represents a market expected to exceed $\$ 9 B$ by 2027 with a growth rate of over $12 \%$. The two systems comprising this technology represent best-in-class portable nuclear magnetic resonance devices. Additional applications include detection of toxins in water supplies, determination of pesticide concentration in runoff, and the useof additives in the oil and gas industry.

\section{BENEFITS}

ERDE provides a best in class compact and portable solution for detection and identification of chemicals in the field. The non-destructive nature and lack of significant background interferences provides exceptional performance in a wide range of operating conditions.

- Ideal for portability

- Both solid and liquid samples can be analyzed

- Exploits a signature space with limited background interference

- Spectra are unique and serve as a fingerprint to identify chemicals

- Demonstrated detection of chemicals through various materials such as non-magnetic pipes

- Small, lightweight, and battery-powered

- Sample measurement is automated and uses small, contained samples

\section{CONTACT}

Amy Migliori

amymigliori@lanl.gov

$505-412-9886$ 
Los Alamos researchers set out to develop sensor to detect chemical weapons agents and quickly realized the breadth of additional applications in the field. The detection of chemical and biological materials in the field provides real-time information to ensure the safety of those exposed, assurance of chemical identity during synthesis, and potential diagnostic applications. This rapid field detection capability ensures accurate identification of rapidly decomposing chemicals that are not amenable to transport to a remote laboratory. It became evident that this sensor had applications far beyond just security or military applications. When there is a need to detect chemicals and biochemical materials in remote locations and the analyses requires a spectrometer, ERDE can detect and identify many classes of chemicals and biological materials quickly and easily without the need to sample the source directly. This in turn is also effectively eliminates the chances of contamination and fouling that is problematic in other devices.

\section{WHAT'S BEHIND OUR TECHNOLOGY}

This technology is different because it uses Earth's magnetic field to create a unique signature space that only emerges under the presence of specific chemicals with specific nuclei in specific spatial arrangements. As such, false positive or false negative data is not generated by different classes of chemicals or materials. Other technologies used in the field suffer from major background interferences and overlapping signatures, making unique identification challenging if not impossible. The Earth's magnetic field ensures a highresolution measurement. By implementing microfluidics sample containment, ERDE has been miniaturized to a portable version that allows for rapid, accurate, and safe identification of small volumes and dilute solutions of hazardous and toxic chemicals and biochemicals.

\section{OUR COMPETITIVE ADVANTAGES}

This technology is the first automated, fluid-based system for the magnetic detection of chemicals and biochemical at Earth's field. In terms of sensitivity, ERDE can detect orders of magnitude lower concentrations than other instruments. ERDE is small enough for field use and can be implemented alongside traditional screening techniques for enhanced chemical and biochemical detection.

\section{OUR TECHNOLOGY STATUS}

Los Alamos researchers have successfully demonstrated this technology in the laboratory on over 40 unique molecules, including pesticides, and have determined that the observed spectra serve as fingerprints for the molecules allowing their structure backprediction. Further, these unique signatures have been measured from chemical warfare agent surrogates, decomposition products, and synthetic precursors. Finally,there is conclusive evidence that common background signatures and potential interferences are invisible to this technology. Los Alamos is seeking qualified licensing or CRADA partners to finalize development of this system.

\section{PUBLICATIONS AND IP}

S133717.001 "Methods and Systems for J-coupled Nuclear Magnetic Resonance" U.S Patent application No. 16/806,862, Appl. Date: 3/2/2020

Kaseman et al. "Design and implementation of a J-coupled spectrometer for multidimensional structure and relaxation detection at low magnetic fields" Rev. Sci. Instr. 91 054103, 2020

Kaseman et al. "Chemical Analysis of Fluorobenzenes via Multinuclear Detection in the Strong Heteronuclear J-Coupling Regime" Appl. Sci. 10(11) 3836, 2020

https://www.lanl.gov/discover/news-release-archive/2021/March/0315-earths-magnetic-field.php 